\title{
The VVDS: a journey through space and time
}

\author{
Garilli, B. ${ }^{1}$, and the VVDS Group \\ ${ }^{1}$ INAF,IASF-Milano, via Bassini 15, 20133, Milano Italy \\ email:bianca@lambrate.inaf.it
}

The VVDS Surveys aim at understanding the combined evolution of galaxies and large scale structure and have by now collected more than 35000 redshifts in four sky areas covering a total of 4.3 square degrees. They are the only surveys of this kind using a purely apparent magnitude selection, thus avoiding the intrinsic biases present in most of the high redshift color based surveys.

The VVDS Deep (0.56 deg $\mathrm{d}^{2}$ down to $I_{A B}=24$, Le Fèvre, et al. (2005)) has already produced milestone results on the evolution of galaxies and large-scale structure up to $z \sim 4$. Most recent results include the mass dependence of the 2-point correlaton function Meneux, et al. (2007): using a mass limited sample selected in the redshift range 0.5-1.2, we detect an increase of the amplitude and slope of $\xi(r)$ with mass. For massive galaxies $(\log \mathrm{M}>=10.5)$, the evolution is essentially in amplitude, while for less massive obejcts (down to $\log (\mathrm{M})=9$ ) there is also an evolution in the shape of the projected function, in the sense of it being flatter at high redshift compared with local Universe. The observed differential evolution indicates a clear trend in the way galaxies are arranged within dark matter halos at different epochs.

The VVDS Wide surveys will cover $10 \mathrm{deg}^{2}$ down to $I_{A B}=22.5$ in four different fields. It aims at reconstructing the complete $3 \mathrm{D}$ maps of the cosmic web up to $z \sim 1$ and at tracing large scale structure up to transverse comoving scales of $100 \mathrm{Mpc} \mathrm{h}^{-1}$ In one of the fields, covering $2 \times 2 \mathrm{deg}^{-2}$ with an average sampling rate of $20 \%$ (Garilli, et al. (2007)) we can probe LSS up to $\mathrm{z}=1.2$. The total number of spectra obtained in this field is 19122, of which 10851 are galaxies, 162 are QSOs and 6801 are stars. The cone diagram shows several structures, with typical sizes of the order of $50 \mathrm{Mpc}$. A prominent clumpy structure at $z \sim 0.52$ extends for more than $100 \mathrm{Mpc}$ and fills the whole transverse dimension of $60 \mathrm{Mpc}$. The higher density regions are generally quite clumpy and there is a strong similarity in the galaxy texture, in the sense that the structures present at $3000 \mathrm{Mpc}$ do look like the ones at $2000 \mathrm{Mpc}$, at least as far as the more luminous galaxies are concerned. This is in agreement with the findings on the evolution of the correlation length (Le Fèvre, et al. (2005)), which is very mild from $\mathrm{z}=1$ to the present epoch. We just note that the distance between the main structures tends to increase as redshift decreases. A detailed study of the correlation function and its dependence on redshift, luminosity, spectral type and mass in this field will be the subject of a paper in preparation.

\section{References}

Garilli,B., et al. 2007, in preparation

Le Fèvre,O., et al. 2005, A\&\&A 439, 845

Le Fèvre,O., et al. 2005, A\&A 439, 877

Meneux,B., et al. 2007, in preparation 